\title{
Is the immediate use of guided bone regeneration on exposed dental implant threads beneficial?
}

\author{
Anwar Ramadhan ${ }^{1 *}$, Abdulaziz Ali ${ }^{1}$, Hessah Aljiran ${ }^{1}$, Kholoud Hasan $^{1}$, John Madda $^{2}$ \\ ${ }^{1}$ Department of Oral and Maxillofacial Surgery, Al-Amiri Hospital, Kuwait City, Kuwait \\ ${ }^{2}$ Department of Pathology, Al-Amiri Hospital Center, Kuwait City, Kuwait \\ Email: ㄹ.
}

Received 23 September 2013; revised 24 October 2013; accepted 31 October 2013

Copyright (C) 2013 Anwar Ramadhan et al. This is an open access article distributed under the Creative Commons Attribution License, which permits unrestricted use, distribution, and reproduction in any medium, provided the original work is properly cited.

\begin{abstract}
Thin buccal bone has a risk of thread exposure, and placement of a graft is the mainline of treatment for thread exposure. However, this process takes time as the patient is expected to wait for healing to occur before the implant can be placed. To overcome the waiting period, this study views a different method. This study examines the use of guided bone regeneration on the day of implant placement on buccal bone where thread exposure occurs. This process has the advantage of avoiding the patient to go through multiple operations till receiving the final prosthetic treatment, also saving time and money for both the patient and the practitioner.
\end{abstract}

Keywords: GBR; Implant; Threads; Exposed

\section{INTRODUCTION}

The effect of becoming partially or totally edentulous can have a severe impact on the social, psychological and functional status, which may cause the patient to feel older, depressed, lose confidence, and have emotional relationship problems. Over the years in dentistry, a variety of options arose to help these patients overcome these complications. Dentures, bridges and more recently dental implants are being used with each having their own sets of advantages and disadvantages. Dental implants are becoming a common choice of treatment in edentulous areas with a success rate of $94 \%-97 \%$ giving the patient a satisfying aesthetic and functional effect [1-5].

As there are numerous advantages to dental implants, there are also disadvantages. Complications can occur when the quality or quantity of jawbone is affected, so bone augmentations have become the standard treatment

*Corresponding author. to create a favorable implant site.

Time needed for maturation of bone after augmentation can take a considerable amount of time before implants can be placed, and thus the question arises if all osseous defects should be treated in this way. The additional time and visits needed are able to affect the cost of the treatment, the time until the final prosthesis is placed, and the amount of surgical intervention needed. And as such reducing this time would be preferable for both the clinician and the patient.

Implant thread exposure is one of the complications that can occur after implant placement due to jawbone anatomy, which can lead to an effect on implant stability, soft tissues, and esthetic.

Treatment of these implants after formation of dehiscence and/or fenestrations with guided bone regeneration (GBR) has proved to be an effective treatment with overall survival rate of the implant of $84.7 \%$ - $100 \%$ with $63 \%-100 \%$ coverage of the defect [6-16].

The basic concept of GBR is to allow space for bone formation by excluding non-osteogenic tissue invasion using a barrier membrane. In this way, the slower proliferating cells from the bone can have adequate time to form into the space.

In this case study, buccal thread exposure that is predicted to happen will be managed with implant placement and GBR at the same visit; bypassing the process of bone augmentation followed by implant placement after bone maturation. Therefore, saving time for both the clinician and the patient will be accomplished.

\section{CASE 1}

A 46 years old female patient, non-smoker, fit and healthy was referred to AL-Amiri Dental Center(Oral and Maxillofacial department) for a bone graft consultation to the 14 region. 


\subsection{Initial Exam}

During the initial clinical and radiological exam (Figure 1), the alveolar ridge showed good vertical height compared to the alveolar crest of the adjacent teeth with approximately $3 \mathrm{~mm}$ reduction in buccal thickness in the 14 region.

The treatment options were discussed with the patient including bone grafts, but the patient decided on having an implant. If thread exposure does occur then guided bone regeneration can be carried out with Implantium (Dentium, South Korea), sizing $12 \mathrm{~mm}$ length $\times 3.6 \mathrm{~mm}$ diameter.

\subsection{Preoperative}

Rinse with chlorhexidine 2\% twice for two days and $2 \mathrm{~g}$ Amoxicillen one hour preoperatively.

\subsection{Operation Procedure}

Alveolar crest incisions were made from the upper right lateral extending to the second premolar. This was followed by a releasing incision distal to the upper right second premolar (Figure 2). A mucoperiosteal flap was

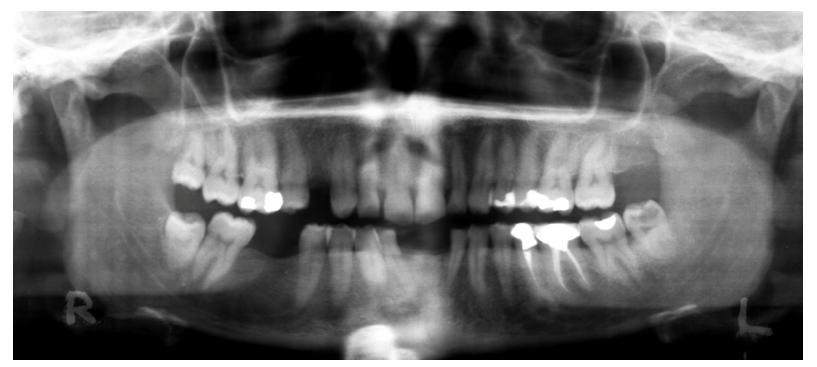

Figure 1. (Case 1) Preoperative panoramic radiography showing the vertical height of the missing upper right first premolar.

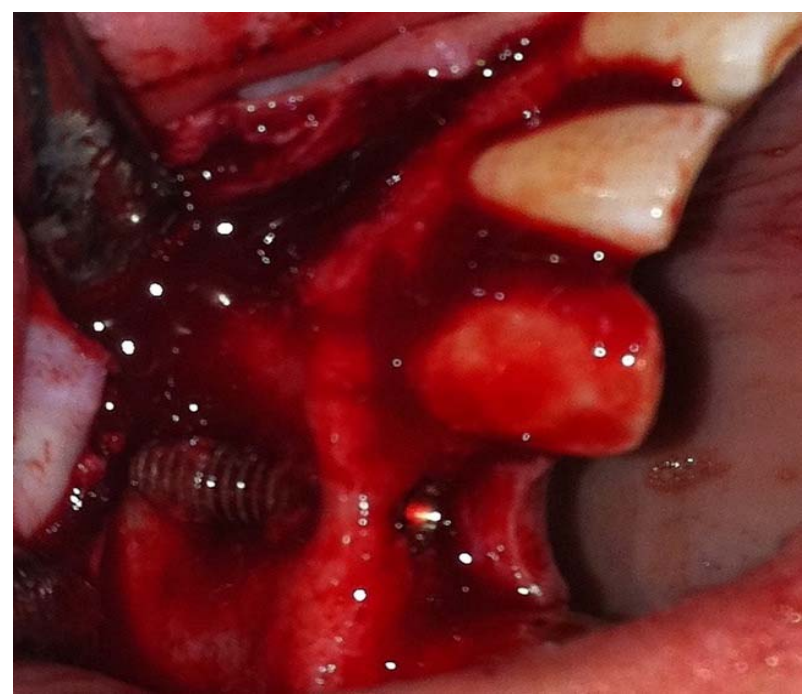

Figure 2. (Case 1) Implant thread buccally exposed at the first premolar region of approximately $8 \mathrm{~mm}$. then raised and followed by implant preparation done according to the Implantium drilling sequence. The implant was manually introduced into bone with $8 \mathrm{~mm}$ thread exposed buccaly. Yet, primary stability was attained (Figure 2).

The exposed thread was covered with allograft bone (TranZgraft: cortical cancellous 0.5 cc; 250 - 1000 microns) followed by a resorbable membrane $10 \mathrm{~mm} \times 20$ mm (Collagen) (Figures 3 and 4).

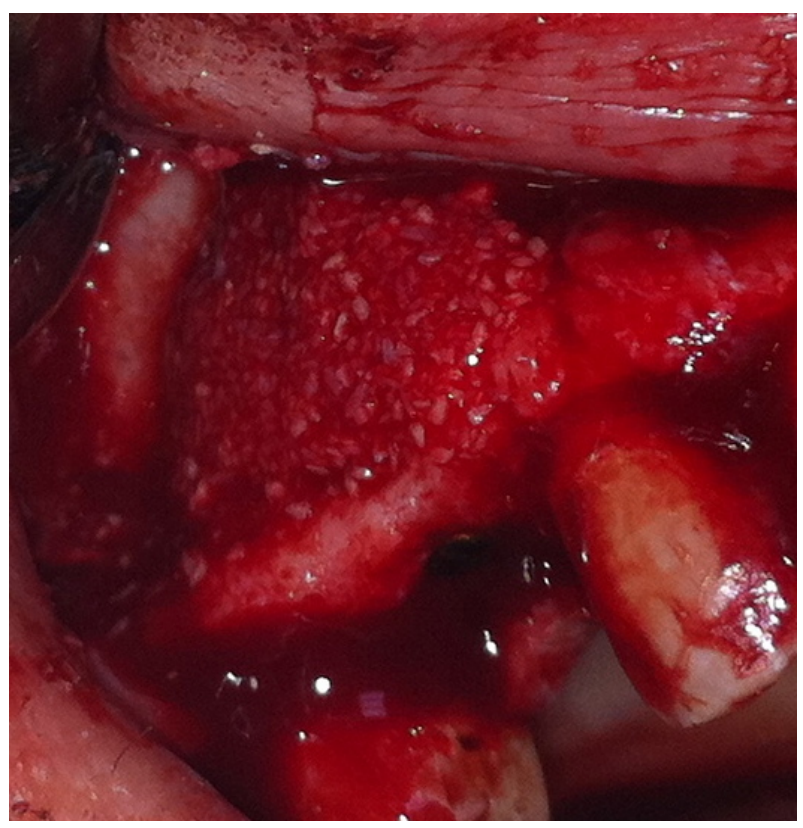

Figure 3. (Case 1) Implant threads covered with allograft bone.

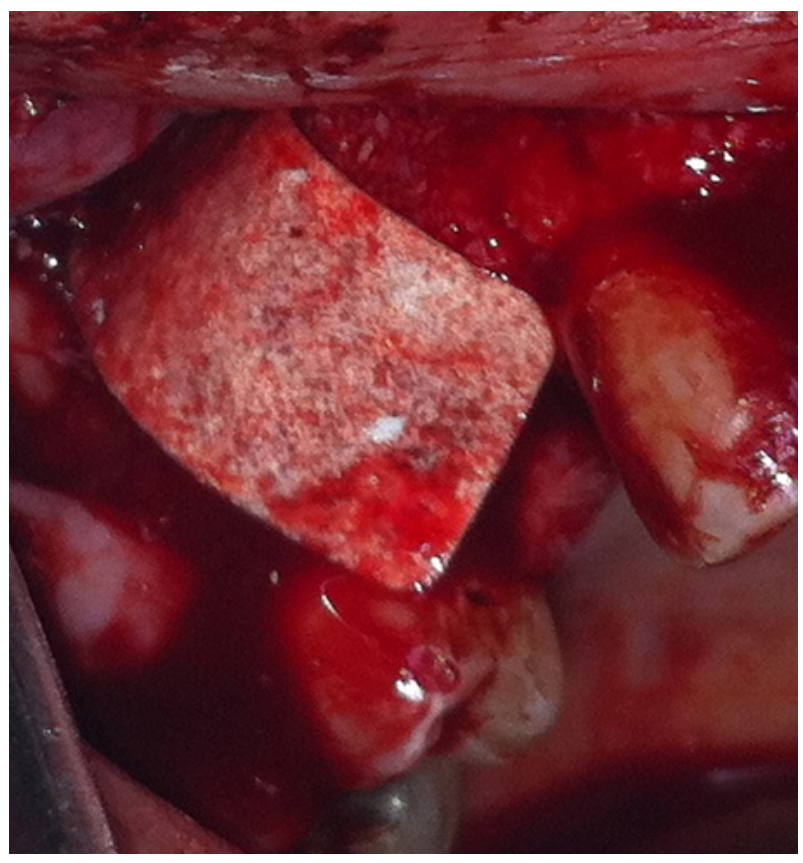

Figure 4. (Case 1) Collagen membrane placed over the allograft bone. 


\subsection{Postoperative}

Amoxicillen $500 \mathrm{mg} 3$ times a day for 7 days was prescribed and the patient was monitored weekly to asses the primary healing for a month. During this period, no abnormal signs or symptoms were reported or observed.

\subsection{Second Stage of Surgery}

After a healing period of three months, the patient returned for placing the healing abutment with no symptoms. Bone graft matured over the cover screw showing extensive bone coverage at the buccal side (Figure 5), and any excess bone was removed and sent to histopathology to investigate the maturation of the bone graft (Figures 6 and 7).

\section{CASE 2}

\subsection{Initial Exam}

A 44 year old female patient attended the surgery

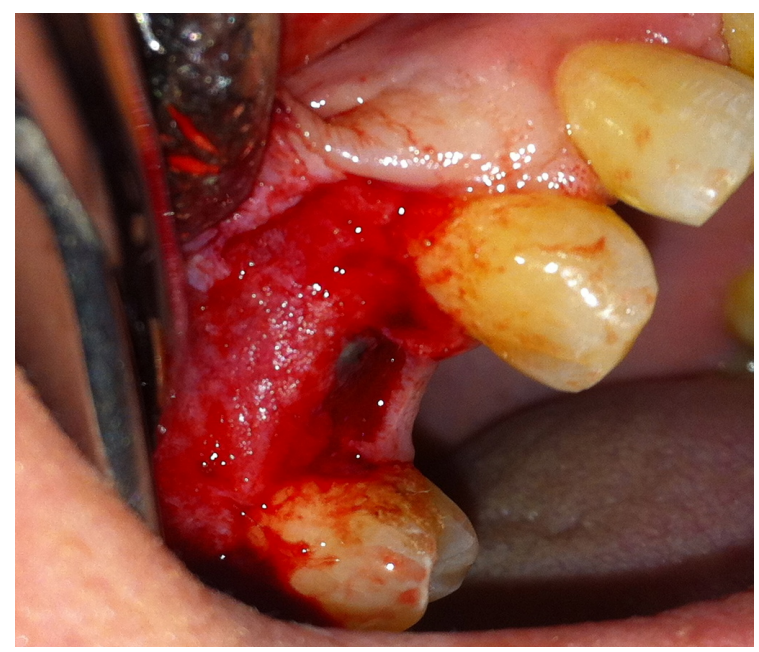

Figure 5. (Case 1) Complete bone healing after 3 months.

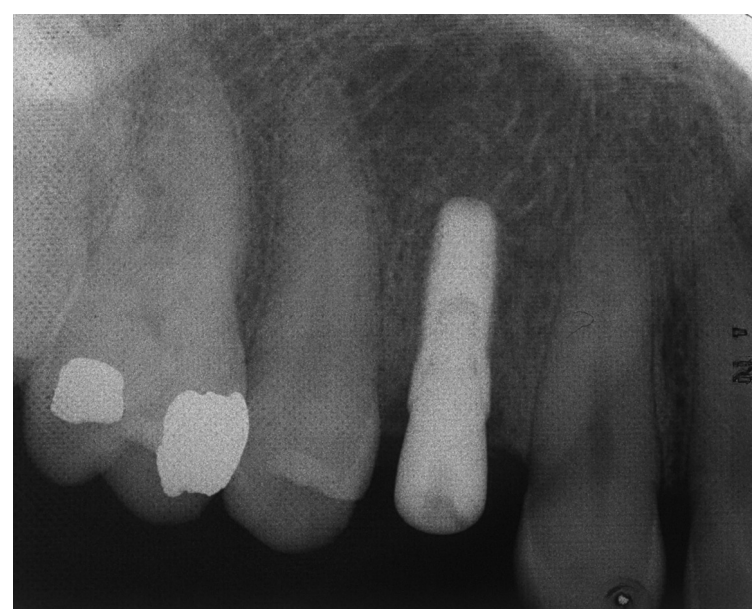

Figure 6. (Case 1) Radiographic follow-up after 3 months.

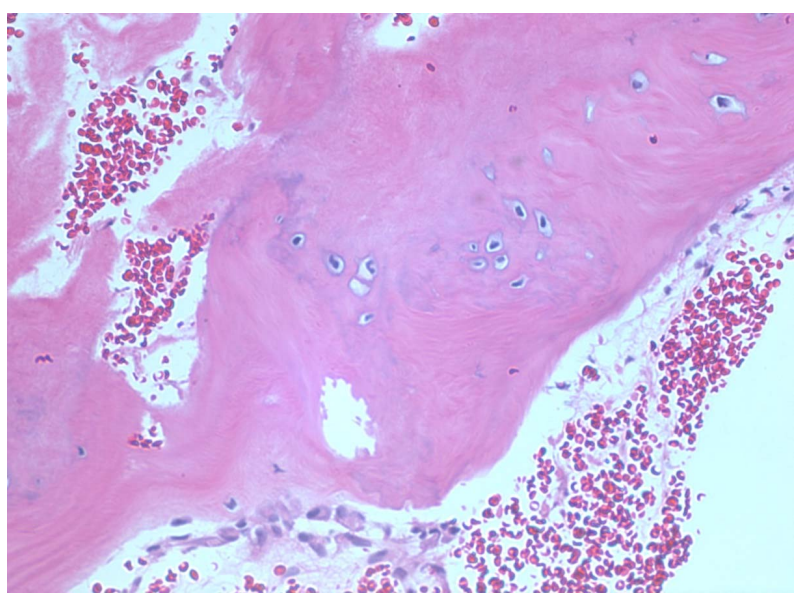

Figure 7. (Case 1) Histological picture of bone removed over cover screw after 3 months during the second stage. H12-2448 mature bone with osteoblasts H\&E X200. Bone was fixed in formalin and decalcified for 30 minutes in acidic solution (TBD-1 Rapid Decalcifier from Thermo Scientific), washed in running tap water before being processed for routine paraffin impregnation and embedding. 4 micron thick section were cut, fixed on glass slides and stained with Haematoxylin and Eosin (H\&E). The sections were examined under transmitted polarized and non-polarized light. Polarized light enhances visualization of bone lamellae. There is a progressive conversion of acellular material (upper left in photo) into mature lamellar bone with osteocytes (bottom right of the trabeculum). Notice the haphazard lamellae in the upper right of less mature bone compared to the lower organized lamellae of a more mature part of bone.

requesting implants in the $15,45,46,47$ regions. Upon clinical and radiographical examination (Figure 8), the patient had insufficient bone thickness at the buccal side. The vertical height in the 15 region was good. The treatment options were discussed with the patient including bone grafts, nonetheless the patient decided on having an implant and if thread exposure does occur then guided bone regeneration can be carried out with Astratech dental implant $10 \mathrm{~mm}$ in length and $3.0 \mathrm{~mm}$ in diameter was planned to be placed.

\subsection{Preoperative}

Rinse with chlorhexidine $2 \%$ twice for two days, and $2 \mathrm{~g}$ amoxicillin one hour preoperatively.

\subsection{Operation Procedure}

An initial alveolar crest incision was made mesial to the upper right first premolar to the right first molar, followed by a releasing incision mesial to the upper right first molar. Mucoperiosteal flap was raised and showed buccal bone defect (Figure 9). Next, an implant preparation was made according to the Astratech dental drilling sequence. The implant was then inserted manually showing $7 \mathrm{~mm}$ of thread exposed buccally with primary 


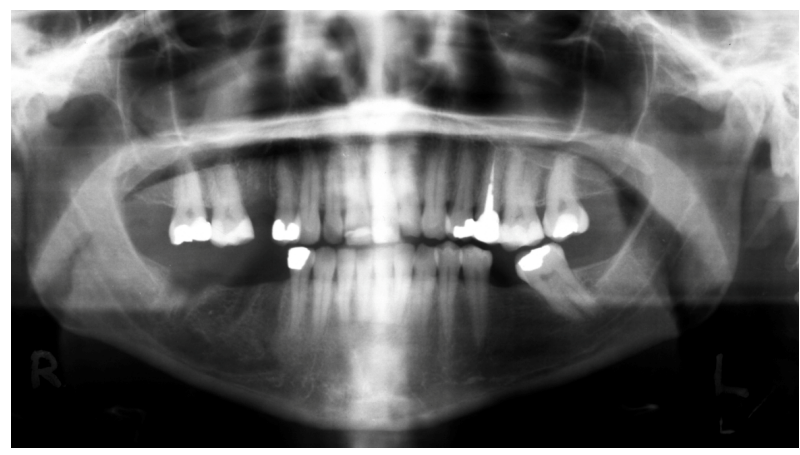

Figure 8. (Case 2) Preoperative panoramic radiography showing the vertical height of the missing upper right second premolar.

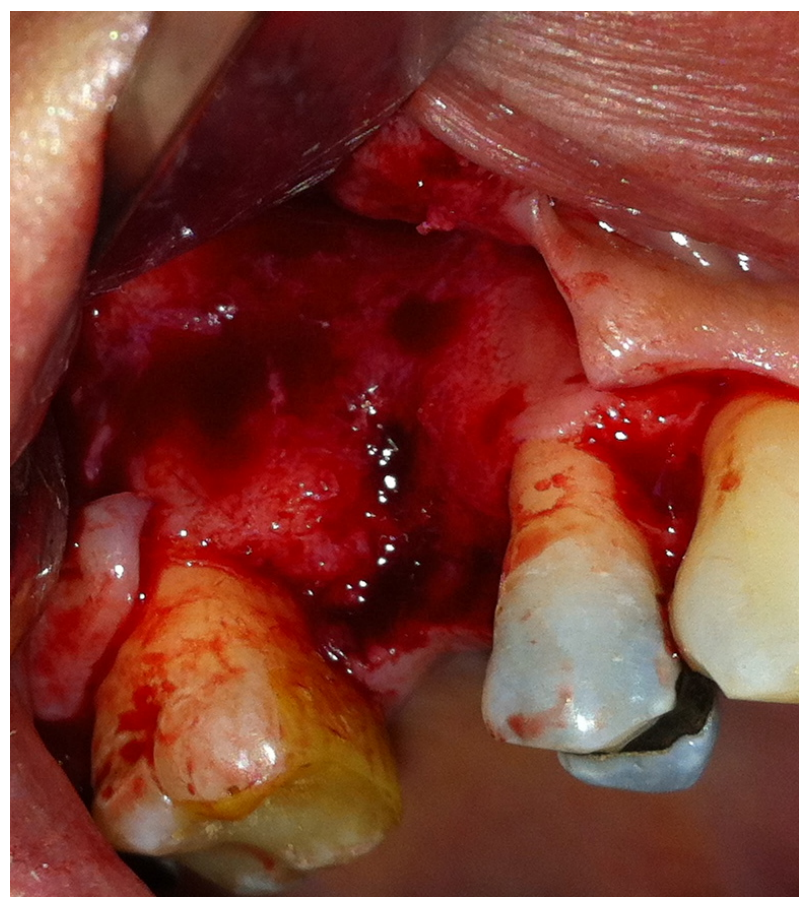

Figure 9. (Case 2) Baccal bone defect after raising the flap.

\section{stability (Figure 10).}

Hence, the thread was covered with allograft bone (TranZgraft: cortical cancellous 0.5cc; 250 - 1000 microns) followed by a resorbable membrane $15 \mathrm{~mm} \times 20$ mm (collagen) (Figures 11 and 12).

\subsection{Postoperative}

Amoxicillen $500 \mathrm{mg}$ was prescribed 3 times a day for 7 days. The patient was followed up on a weekly basis to assess the primary healing for a period of one month. During that period no signs of complications or symptoms were reported.

\subsection{Second Stage Surgery}

Three months later, the patient returned for the placement

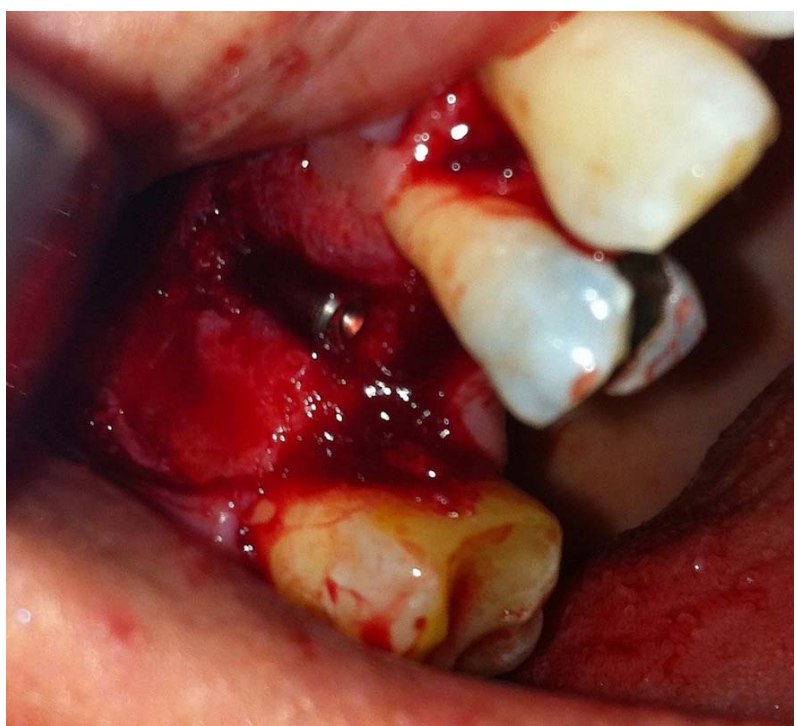

Figure 10. (Case 2) Implant thread exposed buccaly at the second premolar region of approximately $7 \mathrm{~mm}$.

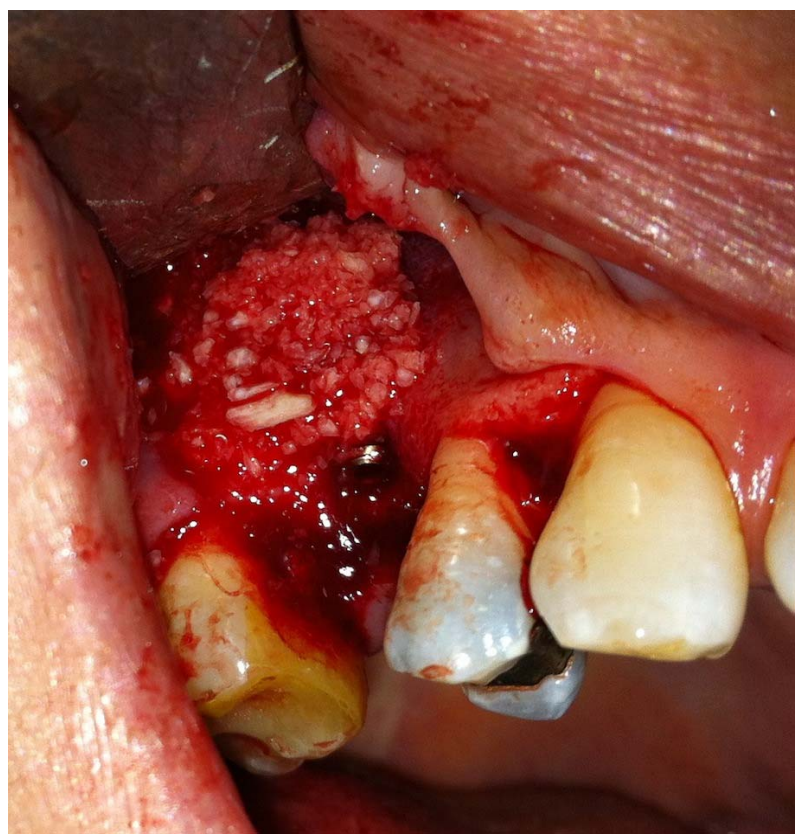

Figure 11. (Case 2) Implant thread covered with allograft bone.

of the healing abutment, and when asked for any symptoms; none were reported. The bone graft extended over the cover screw showing bone coverage at the buccal side (Figures 13 and 14). Excess bone was removed from the buccal side and above the cover screw, and then sent to histopathology to investigate the bone graft maturation (Figure 15).

\section{DISCUSSION}

Two cases were predicted with the risk of threads exposure during clinical examination. Clinically, both showed 


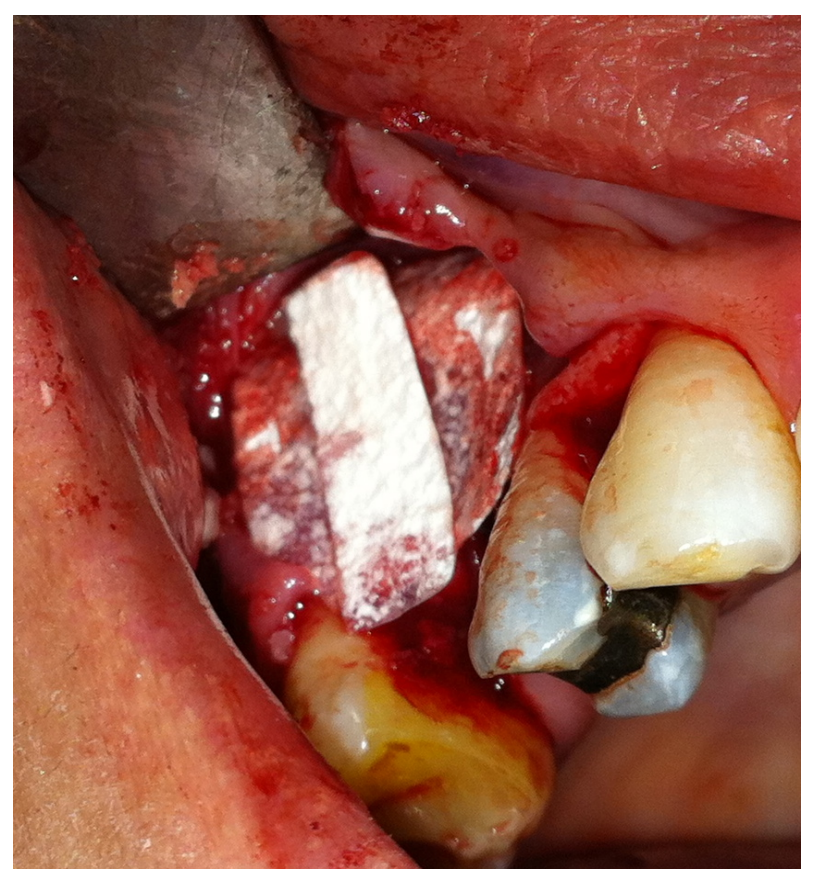

Figure 12. (Case 2) Collagen membrane placed over the allograft bone.

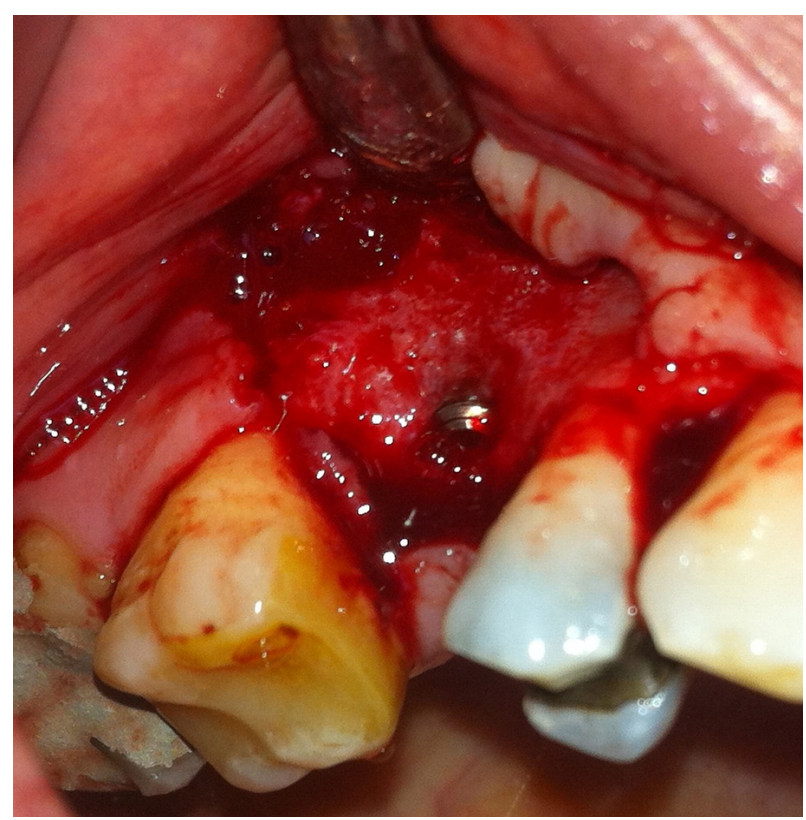

Figure 13. (Case 2) Complete bone healing after 3 months.

thin buccal thickness and patients were informed about the bone graft at the buccal defect followed with implant insertion after bone healing as first option treatment to avoid the risk of thread exposure, and they knew that the treatment would take 6 months until they receive the final prosthetic. Both patients refused the long treatment period. The second option was the GBR. Patients were informed about the risk of success rate. Yet, both patients accepted the second option.

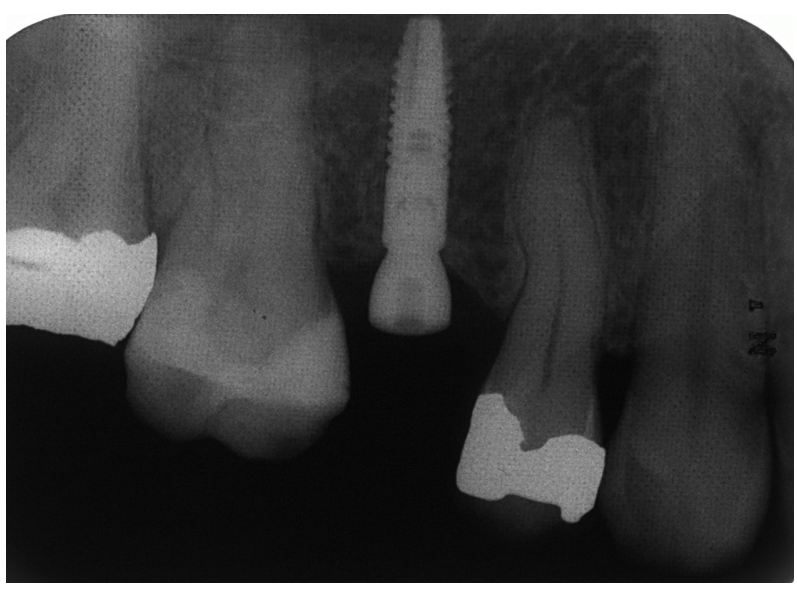

Figure 14. (Case 2) Radiographic follow-up after 3 months.

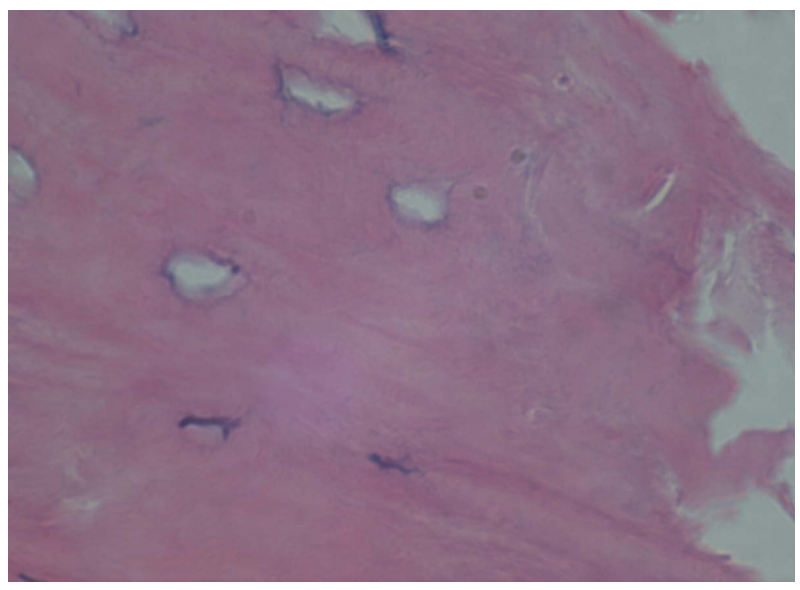

Figure 15. (Case 2) Histological picture of bone removed over cover screw after 3 months during second stageH12-3237 mature bone H\&E X200. Bone was fixed in formalin and decalcified for 30 minutes in acidic solution (TBD-1 Rapid Decalcifier from Thermo Scientific), washed in running tap water before being processed for routine paraffin impregnation and embedding. 4 micron thick section were cut, fixed on glass slides and stained with Haematoxylin and Eosin (H\&E). The sections were examined under transmitted polarized and non-polarized light. Polarized light enhances visualization of bone lamellae. Case 2 shows bone maturation. Haversian canals are scattered throughout the lamellar bone. Note osteocytes around Haversian canals.

During surgical procedure, the vertical threads exposure showed more than $7 \mathrm{~mm}$ on the buccal plate which were reflected in this article (Figures 2 and 10). Exposures were during the insertion of dental implants in the maxilla at the premolar region. The management was immediate GBR with allograft bone followed by collagen membrane.

After three months during the second stage (Figures 5 and 13), Bone integrated over the cover screw in both cases. Therefore, bone was removed and sent for histological evaluation. The histological pictures confirmed the success of bone maturation during 3 months (Figures 


\section{7 and 15).}

Delayed or immediate thread exposure after implant can be managed in multiple ways. These include membrane coverage, and different types of bone grafts (autografts, allografts, alloplasts, or xenografts) [12,17-20].

In 1999, Lorenzoni et al. published a study of 82 patients. They received 85 implants in combination with only expanded polytetrafluroethylene (e-PTFE) for covering the height of bone loss from 2 - $10 \mathrm{~mm}$. After 24 months, the mean bone loss was $1.5 \mathrm{~mm}$ resulting in success. Survival rates in maxilla and mandible in 2 years of all functional implants were 100\% [17].

In 2005, P.A. Fugazzotto reported $98.3 \%$ success rate of 423 implants that were placed in the maxilla and mandible using resorbable tricalcium phosphate or demineralized freeze-dried bone allograft (DFDBA) as GBR technique followed with expanded polytetrafluoroethylene (e-PTFE) in the study [18].

Juodzbalys et al. managed to treat vertical bone loss of 3.8 - $10 \mathrm{~mm}$ defect successfully by simultaneous guided bone regeneration technique with a success rate of $95 \%$ [12].

The survival rate of all functional implants after one to five years was $100 \%$. In the first year, the marginal bone level deficiency was $1.3 \mathrm{~mm}$, and after five years follow up vertical bone loss $2 \mathrm{~mm}$ was demonstrated [12].

Briefly, our studies aim is to reduce the number of surgical procedures and to save time and cost by avoiding the augmentation in the buccal alveolar ridge as a first surgical procedure before inserting the implant after bone augmentation healing.

The success and survival rate of grafting buccal threads exposure in dental implant during the insertion of the implant by guided bone regeneration technique are well-documented [17-19].

According to a recent literature (2012), the success rate of this type of procedure can reach up to $100 \%$ in the maxilla and mandible. On other hand, the survival rate of GBR procedure range from $93.7 \%$ to $100 \%$ in the follow up period of 1 to 11 years [19].

In our report the cases were followed one year, the result showed no resorption of the bone during first year (Figures 16 and 17). On the other hand, the advantages in this study showed short treatment period. Patients received their final prosthetic in three months without any complications by reducing the multiple surgical procedures.

However, the report cannot confirm the success and survival rates as the number of patients were insufficient, but literatures confirmed it with good results by using guided bone regeneration in threads exposure [17-19].

\section{CONCLUSIONS}

This study puts forward that using immediate guided bone

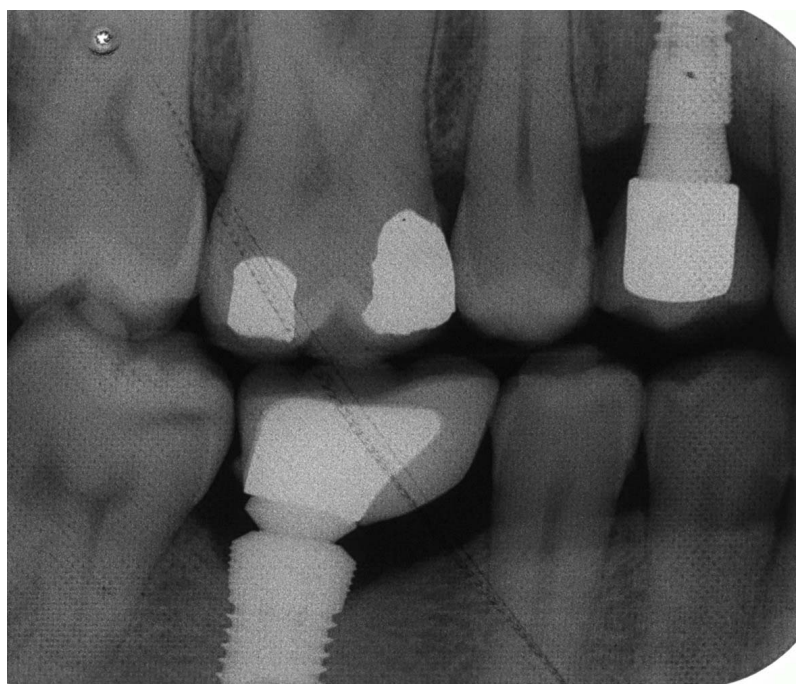

Figure 16. (Case 1) Bitewing $x$-ray taken after one year.

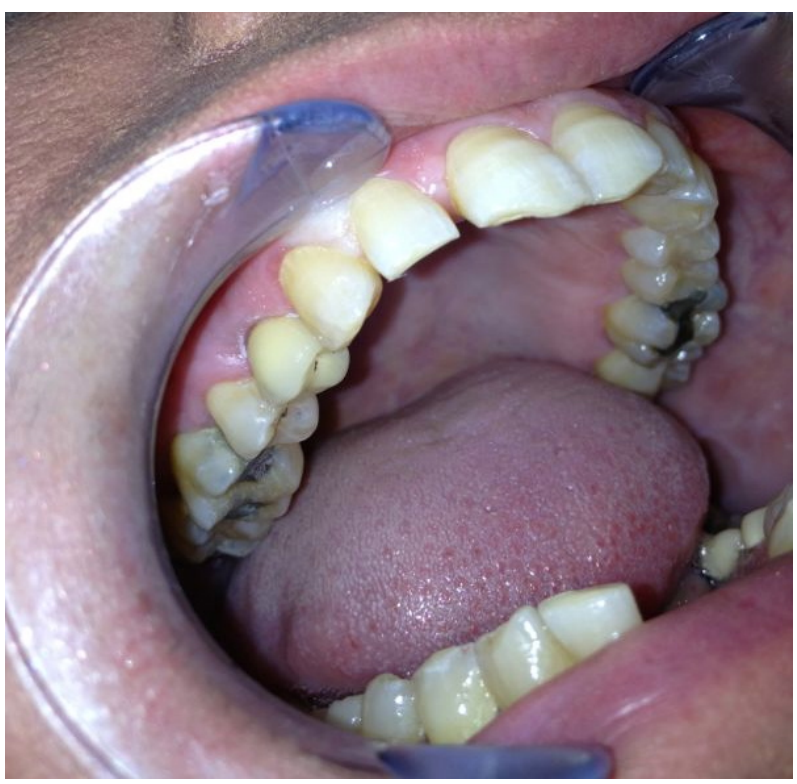

Figure 17. (Case 1) Final prosthetic Result of 14 region after one year functioning.

regeneration over thread exposure at the time of implant insertion has several advantages such as reducing treatment period and saving money.

The success of implant integration with bone is based on primary stability. If you achieve this primary stability and you conceal the implant well, the survival rate will rise.

\section{REFERENCES}

[1] Vigolo, P., Givani, A., Majzoub, Z. and Cordioli, G. (2004) Clinical evaluation of small-diameter implants in single-tooth and multiple-implant restorations: A 7-year retrospective study. International Journal of Oral \& Maxillofacial Implant, 19, 703-709. 
[2] Henry, P.J., Laney, W.R., Jemt, T., Harris, D., Krogh, P.H., Polizzi, G., et al. (1996) Osseointegrated implants for single-tooth replacement: A prospective 5-year multicentre study. International Journal of Oral Maxillofacial Implants, 11, 450-455.

[3] Taylor, R.C., McGlumphy, E.A., Tatakis, D.N. and Beck, F.M. (2004) Radiographic and clinical evaluation of single-tooth biolok implants: A 5-year study. International Journal of Oral \& Maxillofacial Implants, 19, 849-854.

[4] Dhanrajani, P.J. and Al-Rafee, M.A. (2005) Single-tooth implant restorations: A retrospective study. Implant Dentistry, 14, 125-130. http://dx.doi.org/10.1097/01.id.0000163806.14351.54

[5] Fugazzotto, P. A., Beagle, J. R., Ganeles, J., Jaffin, R., Vlassis, J. and Kumar, A. (2004) Success and failure rates of $9 \mathrm{~mm}$ or shorter implants in the replacement of missing maxillary molars when restored with individual crowns: Preliminary results 0 to 84 months in function. A retrospective study. Journal of Periodontology, 75, 327332. http://dx.doi.org/10.1902/jop.2004.75.2.327

[6] Dahlin, C., Lekholm, U., Becker, W., Becker, B., Higuchi, K., Callens, A. and van Steenberghe, D. (1995) Treatment of fenestration and dehiscence bone defects around oral implants using the guided tissue regeneration technique: A prospective multicenter study. International Journal of Oral and Maxillofacial Implants, 10, 312-318.

[7] Zitzmann, N.U., Scharer, P. and Marinello, C.P. (2001) Long-term results of implants treated with guided bone regeneration: A 5-year prospective study. The International Journal of Oral \& Maxillofacial Implants, 16, 355366.

[8] Lorenzoni, M., Pertl, C., Polansky, R.A., Jakse, N. and Wegscheider, W.A. (2002) Evaluation of implants placed with barrier membranes. A restrospective follow-up study up to five years. Clinical Oral Implants Research, 13, 274-280. http://dx.doi.org/10.1034/j.1600-0501.2002.130306.x

[9] Christensen, D.K., Karoussis, I.K., Joss, A., Hammerle, C.H. and Lang, N.P. (2003) Simultaneous or staged installation with guided bone augmentation of transmucosal titanium implants. A 3-year prospective cohort study. Clinical Oral Implants Research, 14, 680-686. http://dx.doi.org/10.1046/j.0905-7161.2003.00963.x

[10] Blanco, J., Alonso, A. and Sanz, M. (2005) Long-term results and survival rate of implants treated with guided bone regeneration: A 5-year case series prospective study. Clinical Oral Implants Research, 16, 294-301. http://dx.doi.org/10.1111/j.1600-0501.2005.01106.x
[11] De Boever, A.L. and De Boever, J.A. (2005) Guided bone regeneration around non-submerged implants in narrow alveolar ridges: A prospective long-term clinical study. Clinical Oral Implants Research, 16, 549-556. http://dx.doi.org/10.1111/j.1600-0501.2005.01154.x

[12] Juodzbalys, G., Raustia, A.M. and Kubilius, R. (2007) A 5-year follow-up study on one-stage implants inserted concomitantly with localized alveolar ridge augmentation. Journal of Oral Rehabilitation, 34, 781-789. http://dx.doi.org/10.1111/j.1365-2842.2006.01679.x

[13] Buser, D., Dahlin, C. and Schenk, R. (1994) Guided bone regeneration in implant dentistry. Quintessence Publishing Co, Inc., Hanover Park.

[14] Lang, N.P., Hammerle, C.H., Bragger, U., Lehmann, B. and Nyman, S.R. (1994) Guided tissue regeneration in jawbone defects prior to implant placement. Clinical Oral Implants Research, 5, 92-97. http://dx.doi.org/10.1034/j.1600-0501.1994.050205.x

[15] Buser, D., Bragger, U., Lang, N.P. and Nyman, S. (1990) Regeneration and enlargement of jaw bone using guided tissue regeneration. Clinical Oral Implants Research, 1, 22-32.

http://dx.doi.org/10.1034/j.1600-0501.1990.010104.x

[16] Nyman, S., Lang, N.P., Buser, D. and Bragger, U. (1990) Bone regeneration adjacent to titanium dental implants using guided tissue regeneration: A report of two cases. International Journal of Oral \& Maxillofacial Implants, 5, 9-14.

[17] Lorenzoni, M., Pertl, C., Polansky, R. and Wegscheider, W. (1999) Guided bone regeneration with barrier membranes: A clinical and radiographic follow-up study after 24 months. Clinical Oral Implants Research, 10, 16-23. http://dx.doi.org/10.1034/j.1600-0501.1999.100103.x

[18] Fugazzotto PA. (2005) Success and failure rates of osseointegrated implants in function in regenerated bone for 72 to 133 months. International Journal of Oral \& Maxillofacial Implants, 20, 77-83.

[19] Clementini M, Morlupi A, Canullo L, Agrestini C, Barlattani A. (2012) Success rate of dental implants inserted in horizontal and vertical guided bone regenerated areas: A systematic review. International Journal of Oral \& Maxillofacial Implants, 41, 847-852. http://dx.doi.org/10.1016/j.ijom.2012.03.016

[20] Jensen, S.S. and Terheyden, H. (2009) Bone augmentation procedures in localized defects in the alveolar ridge: Clinical results with different bone grafts and bone-substitute materials. International Journal of Oral \& Maxillofacial Implants, 24, 218-236. 